\title{
Minimally Invasive Management of Bone Cyst: A case Report
}

\author{
Kumar Anshuman ${ }^{1}$; G S Patnaik ${ }^{2} ;{\mathrm{S} . \mathrm{Nag}^{3}}^{3}$ \\ ${ }^{1 .}$ Assistant Professor Orthopedics ${ }^{2}$ Professor Orthopedics ${ }^{3}$ Professor aand HOD \\ Anesthesia, Narayan Medical college,Hospital Jamuhar, Bihar
}

\begin{abstract}
Bone cysts are benign bone lesions. Various modalities of treatment are described with various techniques with variable results and high recurrence rates. An ideal treatment should be cost effective with minimal morbidity and a short time for bone union and no recurrence. We are presenting a case report of minimally invasive surgical technique for a bone cyst that combines percutaneous decompression and bone grafting.

Key words: Surgical technique, Bone Cyst, Curettage, Autologous bone graft
\end{abstract}

\section{Introduction}

simple bone cysts or unicameral bone cysts are benign cystic lesions of unknown etiology seen in meta-Diaphyseal region of long bones in growing children ${ }^{1}$ Virchow R. described the lesion for the first time in $1876^{2}$ Usually patients are asymptomatic and patient presents usually with a pathological fracture ${ }^{3}$ Various modalities of treatment has been described like open-curettage and bone grafting ${ }^{4}$,intralesional injections of steroids $^{5-6}$,autologous bone marrow ${ }^{7-8}$.A minimally invasive surgical techniquedescribed here preservesperiosteum,muscles and blood supply and provides access to cyst curettage,decompression and use of filling material in this case autologous bone grafts.,gives easy and effective approach and successful outcome.

\section{Case Report.}

A 14 year old was admitted to our hospital with history of recurrent pain and swelling over the right arm. On examination we noted that a swelling 7"x11" fusiform in shape, over the upper arm .There was a past history of trauma and consequent cast application.

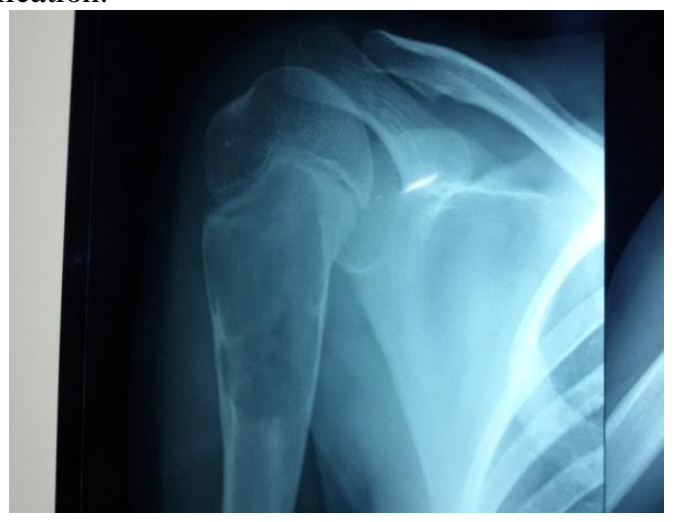

\section{Pre OP X-Ray}

On clinical examination and investigations that included plain $\mathrm{x}$ ray a diagnosis of unicameral bone cyst was provisionally made. FNAC confirmed the diagnosis of unicameral bone cyst.

Surgical Technique 


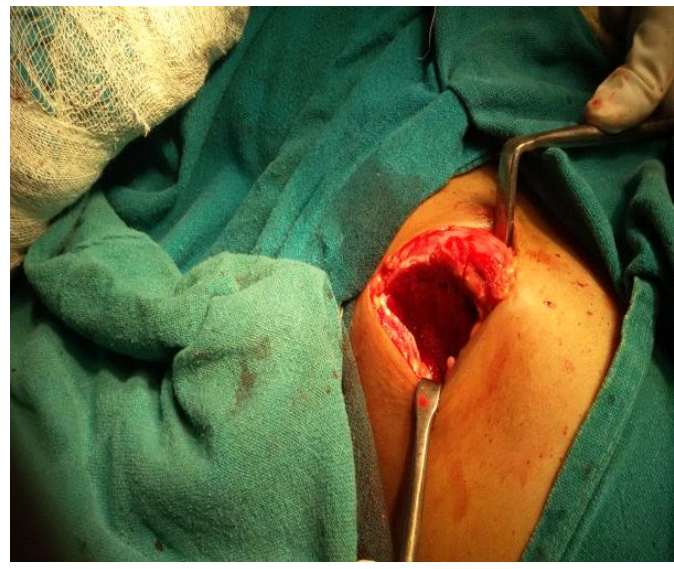

Intra Operative Photo

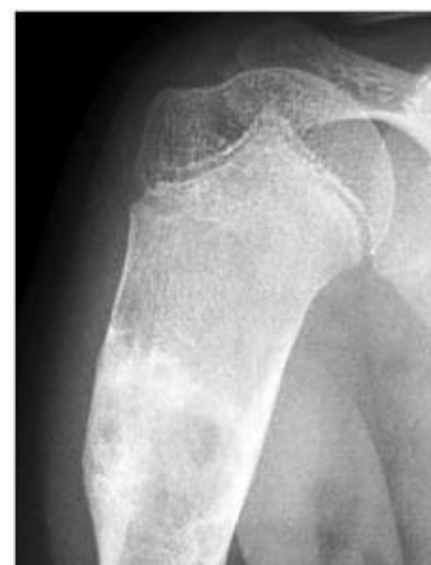

Follow Up X-Ray

Patient was planned for percutaneous cyst curettage and filling of bone cavity with fresh cancellous bone graft under image intensifier guidance. A small longitudinal skin incision ( 2.5 to $3 \mathrm{~cm}$ )was made after localizing the lesion under image intensifier. Bone window was created by fenestration in outer cortex using 3-2 $\mathrm{mm}$ drill bit percutaneously under the guidance of image intensifier. we used an infant feeding tube of size 7 to aspirate the fluid content which was sent for biosy and tissue analysis. Using the tip of a $4 \mathrm{~mm}$ flexible titanic elastic nails the septa. Slivers of iliac crest bone graft thoroughly crushed and were filled in the cavity.

Patient was regularly followed up and subsequent $\mathrm{x}$ rays shows healing of fracture and resolution of lesion and graft integration.

\section{Discussion:}

Cystic bone lesion in first two decades of life constitute common cause for pathological fractures. One has to dwelve with the differential diagnosis of unicameral bone cyst and aneurismal bone cyst.

Percutaneous method under image intensifier guidance using flexible nail for curettage of cyst, decompression along with bone grafting is a treatment option which may be considered with advantage of minimally invasive modality .

\section{References:}

[1]. CohenJ. etiology of simple bone cyst.J Bone Joint Surgery Am 1970;52:1493-97

[2]. Virchow R. On the formation of Bone cysts.In:Uber die bildung von knochencysten berlin: S-B AkadWiss 1876:p369-81

[3]. Campanacci M. CapannaR,PiccinP.unicameral and Aneurysmal bone cysts. ClinOrthop 1986:204:25-36

[4]. Neer CS $2^{\text {nd }}$,Francis KC,MArcoveRC,Terz J, Carbonara PN,Treatment of Unicameral Bone cyst A follow up study of one hundred seventy-five cases jbjs Am 1966:48:731-45

[5]. Oppenheim WL, Galleno H, Operative treatment vs Steroid injections in the management of unicameral bone cysts. J pediatrOrthop 1984;4:1-7

[6]. Hashemi-Nejad A, Cole WG, Incomplete healing of simple bone cysts after steroid injections. J Bone Joint Surg Br 1997;79:72730

[7]. Lokeiz F, Ezra E,Khermosh O, Weintrob S Simple bone cysts treated by percutaneous autologous bone grafting J. Bone Joint Surg Br 1996;78:934-37

[8]. Yandow SM, Lundeen G, Scott SM, Coffin C Autogenic Bone marrow injections as a treatment for simple bone cysts . J paediatrOrthop 1998;18:616-20 